\title{
Estimating the optimal dose of flupentixol decanoate in the maintenance treatment of schizophrenia-a systematic review of the literature
}

\author{
Loren Bailey $^{1}\left(\mathbb{D} \cdot\right.$ David Taylor $^{2}$ \\ Received: 22 October 2018 / Accepted: 11 June 2019/Published online: 12 July 2019 \\ (C) The Author(s) 2019, corrected publication 2019
}

\begin{abstract}
Rationale The licensed dose range for the long-acting injectable antipsychotic flupentixol decanoate (Depixol®) in the treatment of schizophrenia is very broad. This provides little useful direction to prescribers and may ultimately result in patients receiving unnecessarily high doses.

Objectives We aimed to estimate the effect of dose of flupentixol decanoate on relapse rates in schizophrenia and on tolerability by expanding on an earlier review and including non-RCT and German-language studies, as well as using pharmacokinetic and pharmacodynamic data to offer guidance on dosing.

Methods A literature review using EMBASE, Medline, PsycINFO and PubMed was conducted. Treatment success rates at 6 months were extracted or extrapolated from the studies and plotted against dose to estimate a dose-response curve.

Results Data from 16 studies $(n=514)$ allowed estimation of a dose-response curve which rises steeply between the chosen placebo anchor (25\% success rate) and $10 \mathrm{mg}$ every 2 weeks before reaching a maximum between 20 and $40 \mathrm{mg}$ every 2 weeks (80-95\% success rates). Extrapyramidal side effects (EPSEs) were frequently seen (12-71\% of participants) in that dose range. Two -weekly injections seem to provide the highest trough plasma concentration per dose administered and the lowest peak-totrough concentration ratio. Plasma concentration varied up to 5-fold among individuals receiving the same dose.

Conclusions The optimal dose of flupentixol decanoate is likely to be between $20 \mathrm{mg}$ and $40 \mathrm{mg}$ every 2 weeks although higher doses may be required in some individuals owing to variation in drug handling. Doses of flupentixol should be individually established in the range of 10 to $40 \mathrm{mg}$ every 2 weeks according to response and tolerability.
\end{abstract}

Keywords Flupentixol decanoate $\cdot$ Dose-response $\cdot$ Schizophrenia

\section{Introduction}

Flupentixol is a thioxanthine compound licensed for the treatment of schizophrenia and depression. An oral form of the drug was first marketed in Europe in 1965 by Lundbeck. The long-

David Taylor

david.taylor@slam.nhs.uk

Loren Bailey

loren.bailey@slam.nhs.uk

1 Pharmacy Department, South London and Maudsley NHS Foundation Trust, Bethlem Royal Hospital, Monks Orchard Road, Beckenham BR3 3BX, UK

2 Institute of Pharmaceutical Science, King's College London, Fifth Floor, Franklin-Wilkins Building, 150 Stamford Street, London SE1 9NH, UK acting injectable formulation, flupentixol decanoate (Depixol®), followed in 1970. The usual effective dose range for the maintenance treatment of schizophrenia (as specified in the manufacturer's formal summary of product characteristics) "lies between $50 \mathrm{mg}$ every 4 weeks and $300 \mathrm{mg}$ every 2 weeks, but some patients may require up to $400 \mathrm{mg}$ weekly" (Lundbeck Limited 2017a). This represents a 32-fold difference between recommended minimum and maximum dose. The oral formulation has a much narrower recommended dose range of 3 to $18 \mathrm{mg}$ per day - a 6-fold difference. The pharmacokinetic properties of the different formulations are summarised in Box 1 (Lundbeck Limited 2017b; Taylor et al. 2018), but offer no explanation for the wider dose range of flupentixol decanoate. The very broad range of doses recommended for the depot form give little useful direction to prescribers, and the long-acting nature of the formulation makes dose titration very challenging because of the prolonged time to steady-state plasma concentrations. 
Reed and Fanshawe (2011) conducted a review in an attempt to establish a clear dose-response relationship for flupentixol decanoate. They concluded that survival rates (that is the proportion of patients remaining in a study) were highest at a dose of around 50 to $60 \mathrm{mg}$ every 4 weeks. The authors acknowledged that the included studies recruited small numbers and had unusually high survival rates for schizophrenia (above 90\%), suggesting that caution was required when interpreting the results. In addition, the studies reported only average doses and outcomes for groups as a whole, limiting the ability to draw conclusions for individual patients. Included studies were of different lengths ( 24 weeks to 18 months), and the survival rates used to plot the dose-response curve were taken from the end point of the study (i.e. they were not standardised to a specific time period).
In this current review, we aimed to estimate the optimal dose of flupentixol decanoate in the maintenance treatment of schizophrenia and to establish if the evidence from the literature supports the wide dose range specified by the manufacturer. We decided to extend the earlier work by Reed and Fanshawe by including nonrandomised and observational studies (dose finding is not adversely affected by the absence of randomisation or control treatments), and those in the German-language literature. We also aimed to retrieve pharmacokinetic and pharmacodynamic data to provide the theory underpinning any dose recommendations, for example, plasma levels at the minimum effective dose or the relationship between flupentixol dose and dopamine D2 receptor occupancy.

Box 1 Pharmacokinetic data oral vs injection

\begin{tabular}{lll}
\hline & Oral flupentixol dihydrochloride (Depixol®) & Intramuscular flupentixol decanoate (Depixol®) \\
\hline Licensed dose range & $3-9 \mathrm{mg}$ twice daily & 50 mg every 4 weeks to 400 mg every week \\
Bioavailability & $40-55 \%$ & $100 \%$ \\
Time to peak levels & $4 \mathrm{~h}$ & $4-7$ days \\
Mean plasma half-life (SD) & $35 \mathrm{~h}$ & 17 days (9.2) \\
Time to steady state & 7 days & $2-3$ months \\
\hline
\end{tabular}

\section{Method}

In January 2018, a literature review was conducted. EMBASE, Medline, PsycINFO, and PubMed were searched using the terms flupentixol or flupentixol decanoate. Subheadings used were 'adverse drug reaction', 'drug dose', 'drug therapy' and 'pharmacokinetics' in EMBASE and 'administration and dosage', 'adverse effects', 'pharmacokinetics' and 'therapeutic use' in Medline. Additional studies were identified from references. Relevant studies had to include patients with schizophrenia or schizoaffective disorder, use the longacting injectable (LAI) form of flupentixol, be at least 24 weeks in length, and include information on doses used and on relapse or deterioration in mental state as defined by the study. Relevant pharmacokinetic and pharmacodynamic studies had to include information on plasma levels and other relevant data. The search was repeated in January 2019. The Cochrane Systematic Review (Mahapatra et al. 2014) was also reviewed for eligible studies.

Treatment failure (defined as relapse, hospitalisation or clinical deterioration) rates were standardised to 6 months. If 6-month outcome was not reported in the study, the rate was calculated assuming a linear relapse rate as described in Kane et al. (2012). Treatment success rate at 6 months (100 - treatment failure rate as described above) was plotted against mean or fixed dose in milligrams per 2 weeks to estimate a dose-response curve.

\section{Results}

Sixteen studies meeting the inclusion criteria were identified, and eight studies reported on useful pharmacological data (see Fig. 1).

No additional studies of interest were found in the updated search.

The studies provided data on 21 different patient sets with a total of 514 patients and varied in duration from 6 months to 6 years. The study characteristics are displayed in Table 1. All studies included patients with schizophrenia, and the observational studies by Chiliza et al. (2016) and Shajahan et al. (2010) also included patients with schizophreniform disorder, persistent delusional disorder and schizoaffective disorder. Treatment failure was defined as relapse in nine studies (Chiliza et al. 2016; Cookson 1987; Gottfries and Green 1974; Johnson et al. 1987; Kelly et al. 1977; Knights et al. 1979; Laux et al. 2010; Pach et al. 1998; Wistedt 1981), deterioration according to global rating in one study (Agrup-Andersson 
Fig. 1 Study selection process

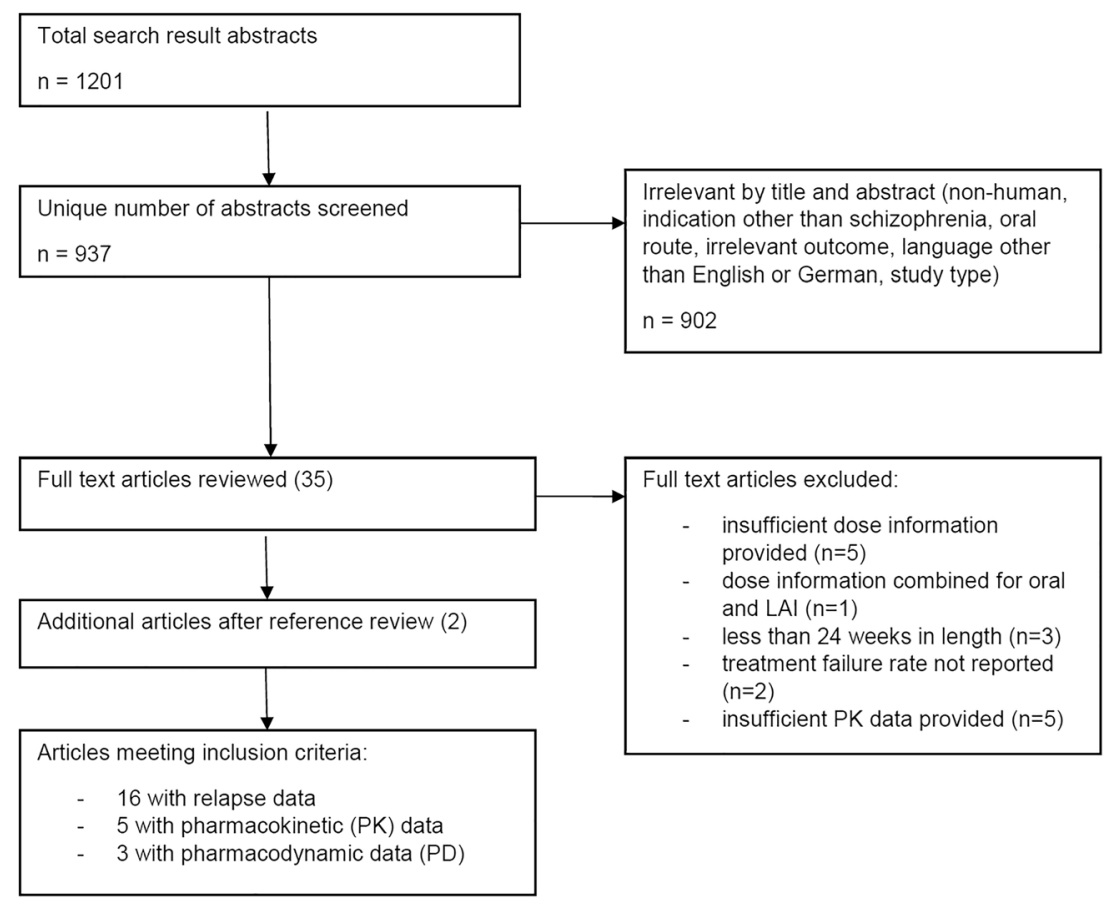

et al. 1974), discontinuation due to poor response in two studies (Dencker et al. 1980; Shajahan et al. 2010), hospitalisation for psychiatric reasons in two studies (Knights et al. 1979; McCreadie et al. 1988), refusal to continue in two studies (Agrup-Andersson et al. 1974; McCreadie et al. 1988) and dropout from trial, not otherwise stated in three (Pinto et al. 1979; Steinert et al. 1986; Wistedt and Ranta 1983).

One study (Dencker et al. 1980) used flupentixol palmitate which has a relative molecular mass (RMM) of $672.92 \mathrm{~g} / \mathrm{mol}$. We converted the doses from this study to flupentixol decanoate (RMM $588.82 \mathrm{~g} / \mathrm{mol}$ ) equivalents using a factor of 0.875 .

\section{Dose-response curve}

The mean doses of flupentixol decanoate ranged from 10 to $333 \mathrm{mg}$ every 2 weeks and averaged $46.2 \mathrm{mg}$ every 2 weeks (see Table 2). Treatment failure rates at exactly 6 months were reported in nine of the studies. For the seven other studies, 6month rates were calculated from the data given using the method described. Treatment success rates standardised to 6 months (100 - treatment failure rate as described above) ranged from 62.5 to $100 \%$ (mean $85.4 \%$ ).

Treatment success rate at 6 months was plotted against mean or fixed dose in milligram per 2 weeks to estimate a dose-response curve (see Fig. 2). The placebo relapse data from Wistedt et al. (1982) was used as an anchor point in the dose-response curve plot.

When limiting the analysis only to studies who defined treatment failure as relapse $(n=530)$, the dose-response curve contains only two points beyond a dose of $40 \mathrm{mg}$ every
2 weeks, but does retain the appearance of a flattening of effect between 20 and $40 \mathrm{mg}$ every 2 weeks. Four studies $(n=74)$ included refusal to continue or drop out for any reason as a measure of treatment failure. All studies used doses of $40 \mathrm{mg}$ every 2 weeks or less, and in one study $(n=31)$, no dropouts were reported. The dose-response curve retains its shape when these studies are excluded.

Seven studies ( $n=386$ ) specifically stated that no additional antipsychotics were allowed during the study period. When limiting the analysis to only these studies, the dose-response curve shows a clear flattening of effect beyond a dose of $30 \mathrm{mg}$ every 2 weeks (see Fig. 3).

One study (Kistrup et al. 1991) was specifically designed to establish the lowest effective dose for flupentixol decanoate in patients with schizophrenia. Patients stabilised on flupentixol decanoate had their dose systematically reduced by one quarter every 12 weeks until symptoms emerged. Eight of the 24 patients tolerated a dose reduction, and the average minimal effective dose was $60 \mathrm{mg}$ every 2 weeks. Eleven patients remained stable on doses of 20 to $40 \mathrm{mg}$ every 2 weeks; seven patients required doses of 50 to $80 \mathrm{mg}$ every 2 weeks; five patients were treated with a dose of $100 \mathrm{mg}$ every 2 weeks, and one person received $250 \mathrm{mg}$ every 2 weeks.

\section{Tolerability}

The proportion of participants experiencing extrapyramidal side effects (EPSEs) and the use of anticholinergic medication were reported in four studies each (see Table 2). We plotted rates of EPSE and rates of anticholinergic prescription against 


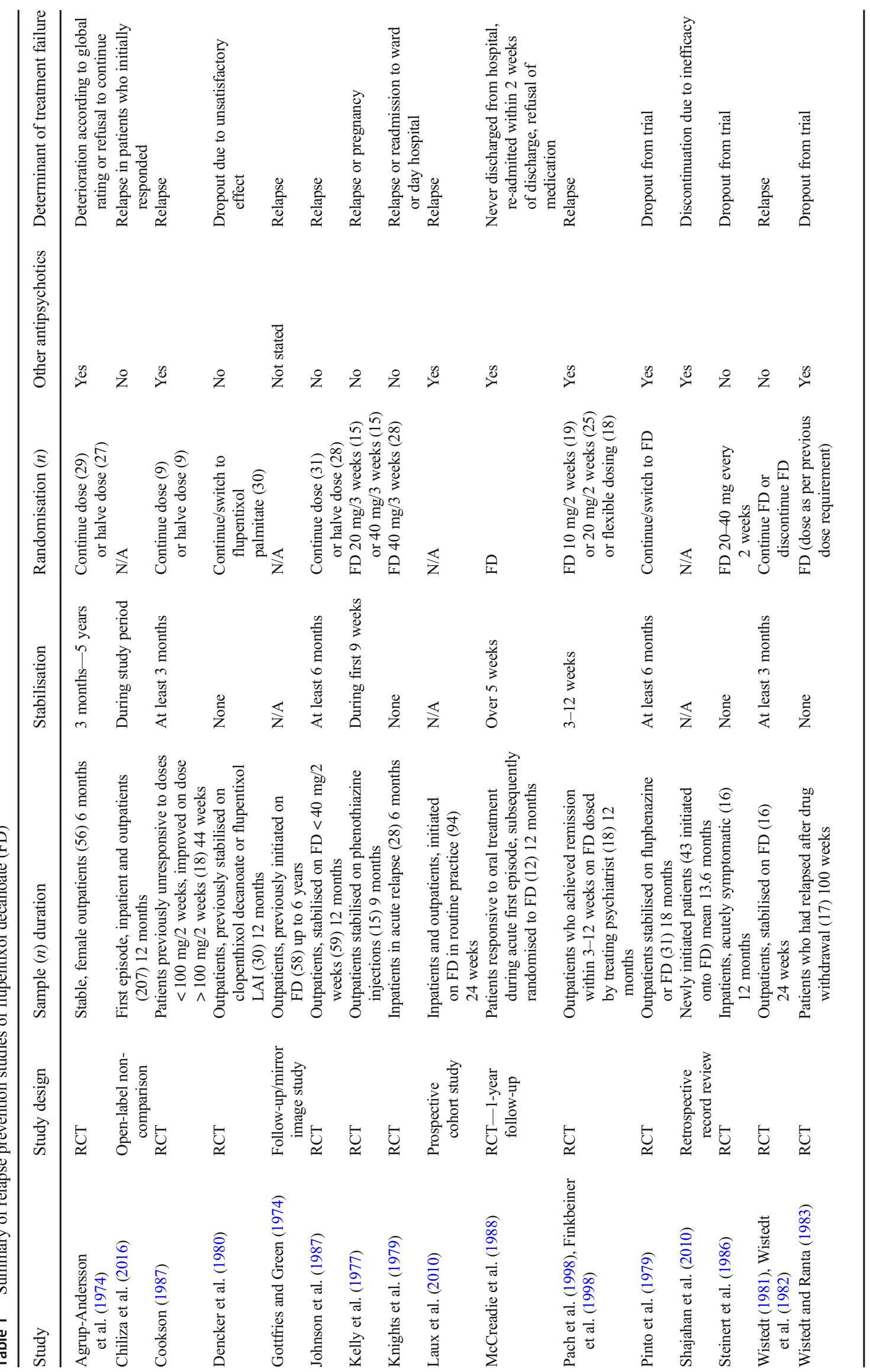




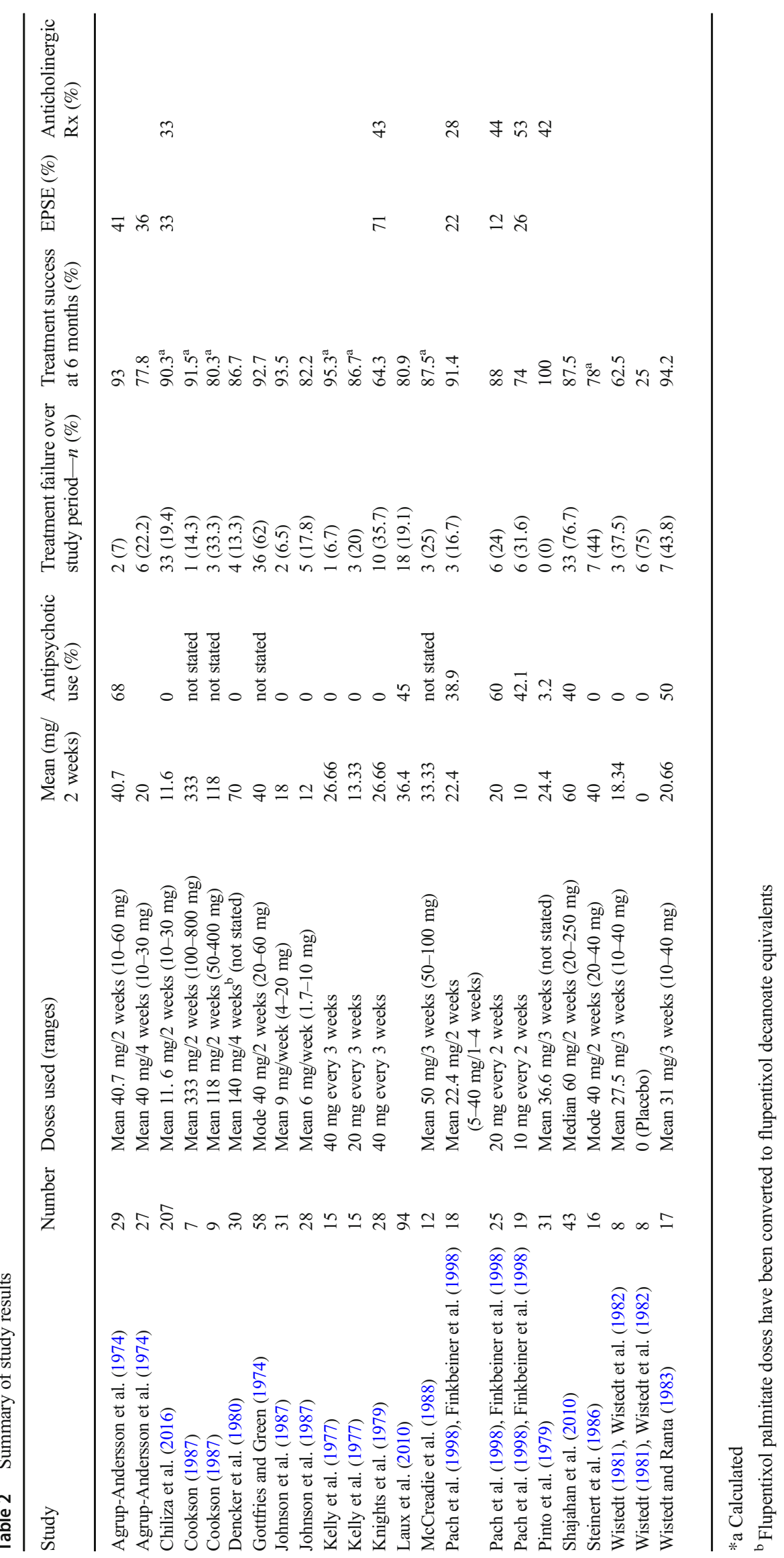


Fig. 2 Dose-response relationship for flupentixol decanoate. Cookson (1987) dose of $333 \mathrm{mg} / 2$ weeks ( $91.5 \%$ success) not included in graph

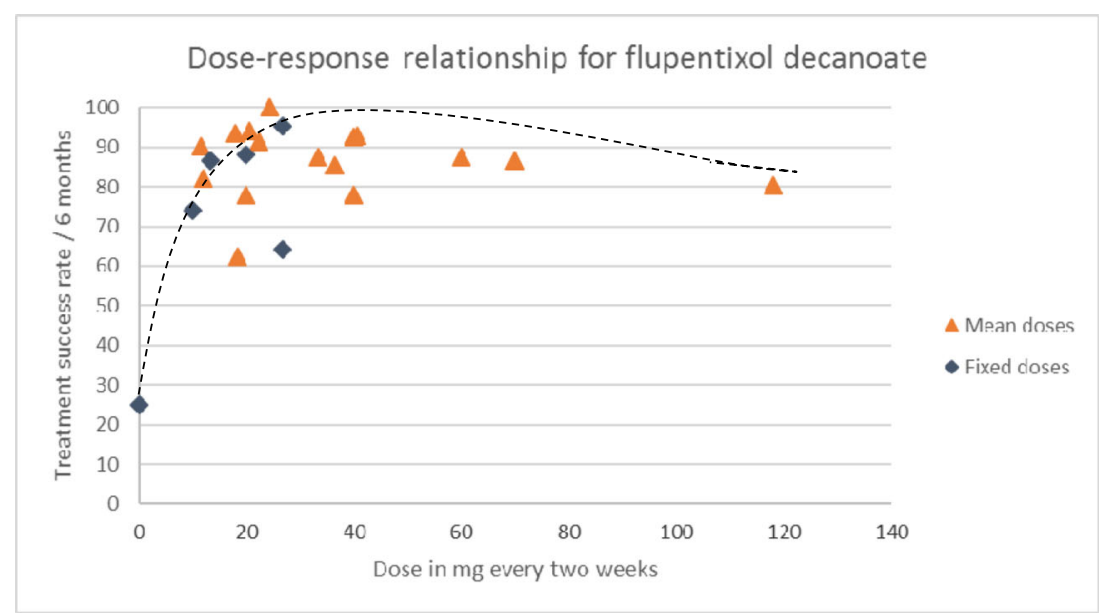

dose (see Figs. 4 and 5). There was no significant correlation between dose and rates of EPSE ( $r=0.386 ; p=0.96)$ or between dose and rates of anticholinergic prescription ( $r=-$ $0.227 ; p=0.46$ ). Extrapyramidal rating scale (EPRS) scores were recorded in two studies (Johnson et al. 1987; Kelly et al. 1977). In Johnson et al. (1987), EPRS scores declined in the half-dose group but remained constant in the full-dose group, and the authors noted a "significant fall in [tardive dyskinesia] during the 6 months following half-dose reduction $(p<0.05)$ ". The study by Kelly and co-workers (1977) found that mean adjusted EPRS scores were non-significantly lower in the $20 \mathrm{mg}$ every 3 weeks group than in the $40 \mathrm{mg}$ every 3 weeks group ( 0.32 and 1.44 respectively). Discontinuation owing to side effects was reported in five studies and ranged from $0 \%$ (Steinert et al. 1986) to 1\% (Chiliza et al. 2016), 4\% (Gottfries and Green 1974) and 25\% (Laux et al. 2010) to $37 \%$ (Shajahan et al. 2010).

\section{Pharmacokinetics and pharmacodynamics}

Pre-injection ('trough') flupentixol serum levels for individual subjects were reported in five studies (Cookson 1987; Jørgensen and Overø 1980; Kistrup et al. 1991; Saikia and Jørgensen 1983; Turbott et al. 1985), providing data for a total of 95 patients (Table 3 ).

The studies used different assay methods to estimate serum concentrations of cis-flupentixol with levels per dose administered ranging from 0.01 to $1.8 \mathrm{ng} / \mathrm{ml}$ per $\mathrm{mg} /$ week. The study by Kistrup et al. (1991), which used high-performance liquid chromatography, reported a higher average serum level per dose administered than the other studies (see Table 3). Jørgensen and Overø (1980) used flupentixol palmitate and normalised all serum levels to a dose of $100 \mathrm{mg}$ every 4 weeks. In the study by Turbott et al. (1985), serum levels were expressed as haloperidol equivalents. Because of this
Fig. 3 Dose-response relationship for flupentixol decanoate. Studies allowing additional antipsychotics have been excluded

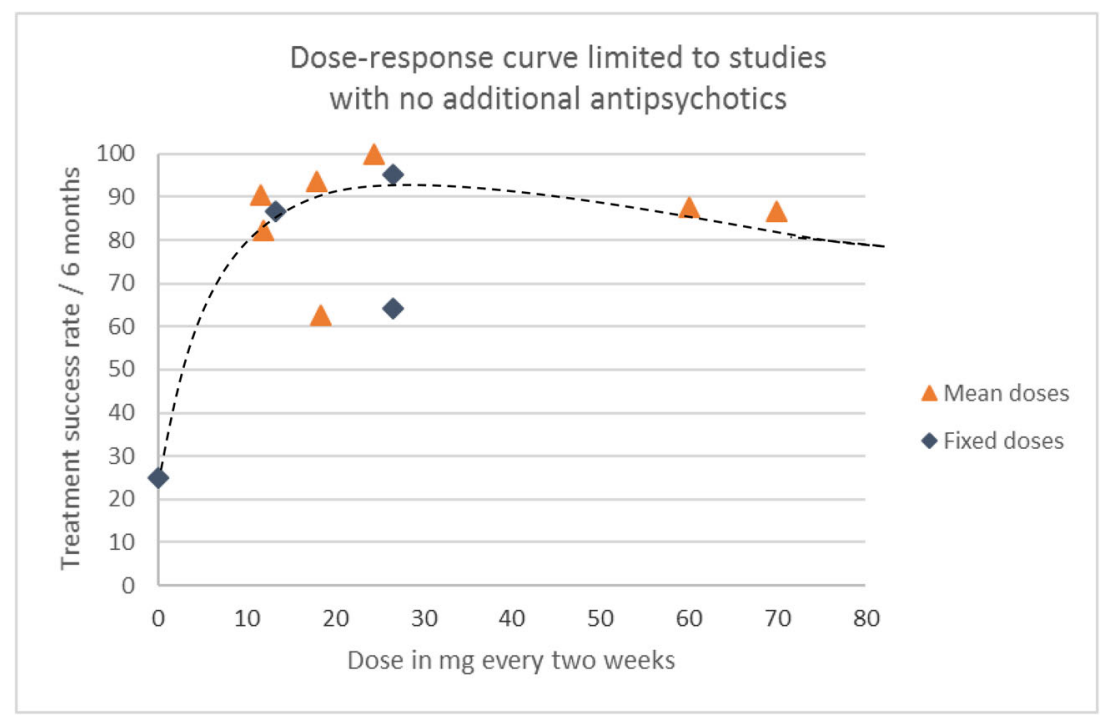




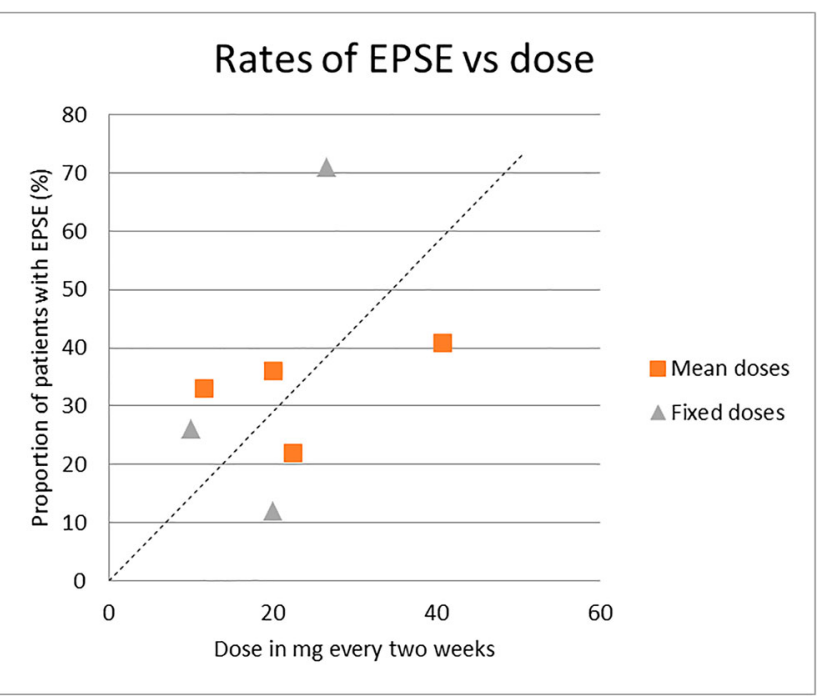

Fig. 4 Rates of EPSE vs dose. The dotted line represents the line of best fit

heterogeneity in the assaying and reporting of serum levels, it was not sensible to consider these results together.

The study by Jørgensen et al. (1980) found serum levels to peak 7 days after the injection was administered, after which they declined with an average apparent half-life of 17 days. The ratio of peak to trough (pre-injection) serum levels varied from 1.6 to 10.4 , a 6.5 -fold variability in apparent half-lives between patients. Similar doses produced serum levels differing up to 5-fold for peak levels and 3-fold for trough levels. For example, in the 11 patients who received $100 \mathrm{mg}$ every 4 weeks, trough serum levels varied from 1.1 to $3.0 \mathrm{ng} / \mathrm{ml}$ and peak levels varied from 2.9 to $14.7 \mathrm{ng} / \mathrm{ml}$.

Saikia and Jørgensen (1983) also found an approximate 3fold variation in the trough serum levels of patients receiving the same dose. For example, the five patients receiving $20 \mathrm{mg}$ every 2 weeks had pre-injection serum levels ranging from 1.4

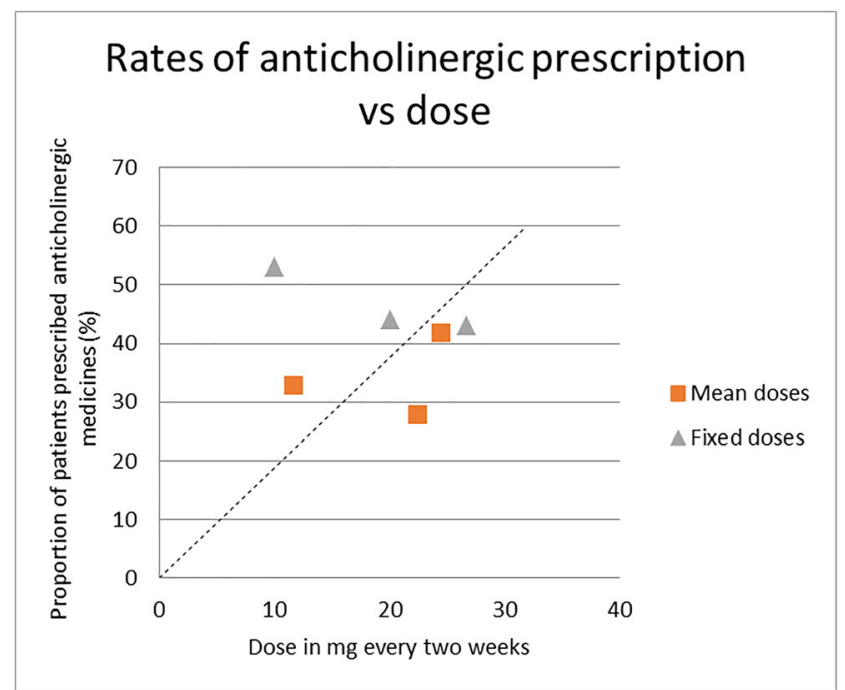

Fig. 5 Rates of anticholinergic prescription vs dose. The dotted line represents the line of best fit to $4.1 \mathrm{ng} / \mathrm{ml}$. The average peak-to-trough level ratio was lowest in the two weekly group at 1.7 , compared with 2.9 in the three weekly group and 2.6 in four weekly group. Variability of this ratio between patients was 2-fold in the two weekly group ( $n=13), 3$-fold in the three weekly group $(n=4)$ and 5 fold in the four weekly group $(n=6)$.

Three pharmacodynamic studies were identified (BalantGorgia et al. 1985; Farde et al. 1989; Reimold et al. 2007), all in patients taking oral flupentixol. A study by BalantGorgia et al. (1985) suggested that a plasma level of $2 \mathrm{ng} / \mathrm{ml}$ is required for clinical response (defined as 50\% improvement in BPRS score over a minimum of 10 days). Doses ranged from 6 to $12 \mathrm{mg}$ /day and steady-state plasma levels from 1.2 to $5.2 \mathrm{ng} / \mathrm{ml}$. In a study by Reimold and co-workers (2007), therapeutic doses led to D2 receptor occupancies (D2-RO) between 50 and $70 \%$, with a corresponding $\mathrm{ED}_{50}$ of $0.68 \mathrm{ng} / \mathrm{ml}$. No patient's plasma concentrations exceeded $2 \mathrm{ng} / \mathrm{ml}$, and no D2-RO was higher than $70 \%$.

\section{Discussion}

\section{Dose-response curve}

The dose-response curve rises steeply between the placebo anchor and $10 \mathrm{mg}$ every 2 weeks before reaching a maximum between 20 and $40 \mathrm{mg}$ every 2 weeks. At doses above $40 \mathrm{mg}$ every 2 weeks, the curve begins to flatten and then fall, with higher doses seemingly producing lower treatment success rates.

For antipsychotics in general, the dose-response curve usually resembles an S shape (Davis and Chen 2004): at very low doses, response is minimal until a threshold dose is reached. Around this latter dose, the response curve rises steeply (almost vertically if not plotted logarithmically) before flattening off to a maximum, with dose increases producing no further effect. This characteristic pattern might be thought of as being analogous to a light switch - the drug is either 'on' of 'off' according to its dose. For long-acting injectable antipsychotics, this dose-response pattern has been demonstrated for haloperidol decanoate (Taylor 2005) with the dose of maximal effect estimated to be, at most, $100 \mathrm{mg}$ every 4 weeks. It can also be seen with paliperidone palmitate and risperidone long-acting injections (Kane et al. 2003; Pandina et al. 2010).

The studies reviewed here are unable to clearly demonstrate a precise threshold dose of flupentixol decanoateeven doses of $10 \mathrm{mg}$ every 2 weeks produced treatment success rates far exceeding those of placebo (although it should be noted that the position of the placebo anchor is derived from a single study). Other available estimates of the placebo effect are higher, e.g. 36\% (Leucht et al. 2012) and 60\% (Kane et al. 2012), but still lower than the effect of $10 \mathrm{mg}$ every 2 weeks. 


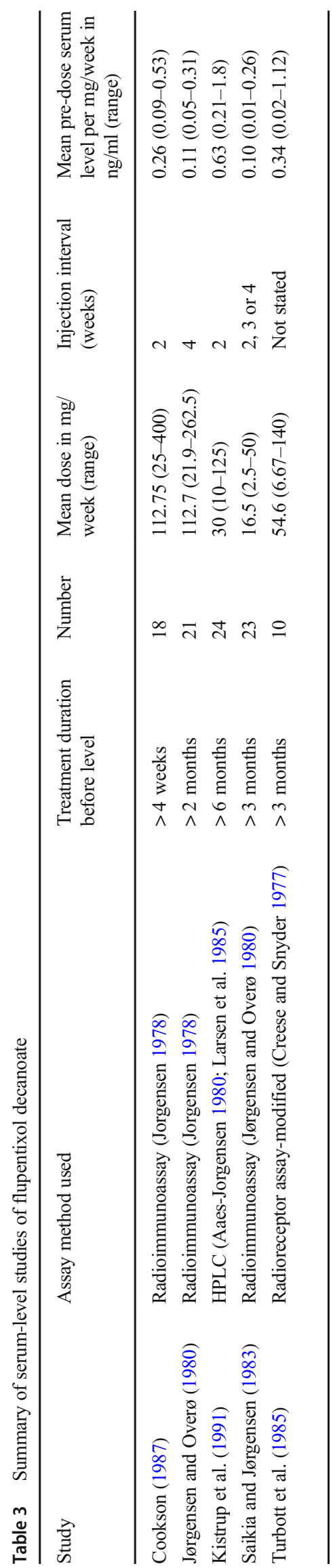

The dose-response curve suggests that $40 \mathrm{mg}$ every 2 weeks may be the dose of maximal effect. The curve of the doseresponse plot not only stops rising beyond this dose, it actually appears to decline, suggesting that higher doses may be somewhat detrimental to therapeutic effect (as seen with oral quetiapine (Arvanitis and Miller 1997) and oral risperidone (Ezewuzie and Taylor 2006)). The data at this end of the response curve, however, are mean doses, rather than fixed doses, and therefore contain a range of doses. The apparent decline in the curve in this dose range may be an artefact of ever-increasing doses for treatment-resistant patients (who were unlikely to respond to any dose). However, doses in the naturalistic or flexible dose studies rarely exceeded $60 \mathrm{mg}$ every 2 weeks, suggesting that levels of treatment resistance were low. The study by Cookson (1987), which used the highest doses, included only treatment-responsive patients. Adding results from this study to the graph produces a marked flattening of the curve.

When only the fixed dose, relapse prevention studies were included, the curve shows no plateau, but stops at $26.66 \mathrm{mg}$ every 2 weeks, thus limiting the ability to draw conclusions about higher doses and their possible effect.

Many of the studies were from times where additional antipsychotic use was a common practice. However, three quarters of the total sample came from studies where additional antipsychotics were not permitted. This allows reasonable estimation of dose-response for flupentixol decanoate without the confounding effects of other antipsychotic use. When including only these single-drug studies, the dose-response curve retains its $\mathrm{S}$ shape with a plateau around $30 \mathrm{mg}$ every 2 weeks.

Studies in which the outcome was dropout for any reason were included, because often poor clinical effect is a reason for stopping medication (Lieberman et al. 2005; Taylor et al. 2006). The numbers of participants in these studies were small and did not alter the shape of the dose-response curve.

\section{Dose ranges used in the literature}

Doses reported in the studies ranged from $1.7 \mathrm{mg}$ per week to $800 \mathrm{mg}$ every 2 weeks. We found evidence that a dose as low as $10 \mathrm{mg}$ every 2 weeks was effective at preventing relapses in the medium term, and no evidence to support use of doses even approaching the highest UK recommended dose (400 mg/week).

The effective doses found here are consistent with the findings from other dose comparison RCTs. In their meta-analysis of relapse prevention studies, Uchida et al. (2011) find that low doses of antipsychotics [0.5-1 times the defined daily dose (DDD)] were as effective as standard doses (1 DDD or greater), whereas very low doses (less than 0.5 DDD) were inferior. The DDD for intramuscular flupentixol decanoate is $4 \mathrm{mg} /$ day, equating to $56 \mathrm{mg}$ every 2 weeks. Our results 
suggest that a dose of 0.35-0.7 DDDs is optimally effective at preventing relapses. As defined by the World Health Organisation (2018), the DDD is "the assumed average maintenance dose per day for a drug used for its main indication in adults", not necessarily the recommended or most commonly prescribed dose.

In the UK and Ireland, flupentixol decanoate is marketed as Depixol® injection, and specifies a licensed dose range of $50 \mathrm{mg}$ every 4 weeks up to a maximum of $400 \mathrm{mg}$ every week for the maintenance treatment of schizophrenia. Our review suggests that doses beyond $40 \mathrm{mg}$ every 2 weeks are unlikely to produce further beneficial effects for most patients. In other countries, e.g. Australia, Canada, France and New Zealand, flupentixol decanoate is marketed as Fluanxol® injection. The recommended dose range as stated in the product labels is 20$40 \mathrm{mg}$ every 2 to 4 weeks in Australia and New Zealand, 20$40 \mathrm{mg}$ every 2 to 3 weeks in Canada and 20-80 mg every 2 weeks in France. The Canadian product label also states that doses above $80 \mathrm{mg}$ are rarely necessary but may be considered for individual patients. It is hard to explain why the dose ranges should differ so greatly between two versions of the same active ingredient produced by the same pharmaceutical company.

\section{Lowest effective dose}

The study by Kistrup et al. (1991) demonstrated that for just under half of the studied patients, the lowest effective dose was between 20 and $40 \mathrm{mg}$ every 2 weeks. For a further $30 \%$, doses up to $80 \mathrm{mg}$ every 2 weeks were sufficient, and only a quarter of patients apparently needed doses above $80 \mathrm{mg}$ every 2 weeks.

The three dose reduction studies (Agrup-Andersson et al. 1974; Cookson 1987; Johnson et al. 1987) found higher relapse rates for previously stable patients who had their dose reduced by half compared with those who remained on their stable dose. The doses these patients had been stabilised on differed in each study, with one study only including patients stabilised on doses lower than $40 \mathrm{mg}$ every 2 weeks and one study including only patients who had responded to doses above $100 \mathrm{mg}$ every 2 weeks but not to lower doses. The third study, which allowed flexible dosing between 10 and $60 \mathrm{mg}$ every 2 weeks prior to dose reduction, was the only one that found no statistically significant difference in relapse rates. These varied findings perhaps reflect the large differences in PK characteristics between individuals. Increased rates of relapse on dose reduction may also reflect dopamine supersensitivity psychosis (Chouinard et al. 1978).

In receptor occupancy studies, therapeutic doses of oral flupentixol led to D2-RO between 50 and $70 \%$, with a corresponding $\mathrm{ED}_{50}$ of $0.68 \mathrm{ng} / \mathrm{ml}$. No studies investigated D2-ROs for patients treated with flupentixol decanoate, and therefore, we cannot draw conclusions about the lowest dose of flupentixol decanoate that would lead to therapeutic D2-ROs.

\section{Maximum effective dose}

None of the included studies were designed to identify a dose beyond which no further therapeutic response would be seen. The study that used the highest doses of flupentixol decanoate (Cookson 1987) did so for a subset of patients who responded only to dose increases beyond $100 \mathrm{mg}$ every 2 weeks, when lower doses had been ineffective. Relapses were more frequent in those subjects who then had their dose reduced by half. This is perhaps the most compelling evidence that higher doses may be required in some individuals.

The highest dose administered in fixed dose studies was $26.66 \mathrm{mg}$ every 2 weeks and producing a treatment success rate of $95 \%$ over 6 months in a relapse prevention study and $64 \%$ in an acute treatment study.

The receptor binding studies used oral flupentixol and did not find doses or serum levels at which saturation or near saturation of dopamine receptors occurs. Thus, a plateau of effect cannot be predicted from these studies.

\section{Dosing interval}

No study investigated the effect of dosing interval on treatment failure. Treatment success rates were similar when grouped according to injection interval. The pharmacokinetic data suggests that two weekly injections provide the highest trough plasma level per dose administered and less fluctuation of serum levels within an individual. The apparent half-life of 17 days would also support an injection interval of 2 weeks. Only one study (Pach et al. 1998) specifically allowed an injection interval of 1 week, but it is unclear how many patients received their injection weekly. The time to maximal plasma concentration is 7 days, suggesting that weekly dosing is unnecessary unless a large volume of injection is to be given. Injection intervals of more than 4 weeks were not studied.

\section{Tolerability}

One to two thirds of patients in the included studies experienced EPSE, although the exact number was dependent on the dose they received - severity of EPSE and incidence of tardive dyskinesia reduced with lower doses. However, doses below $10 \mathrm{mg}$ every 2 weeks were not studied with respect to EPSE or therapeutic effect. The lack of a defined threshold dose for therapeutic effect makes it impossible to say with any certainty whether EPSE are always present in therapeutic use, that is, an inevitable consequence of therapeutic dosing.

Flupentixol has occasionally been described as a partial atypical antipsychotic due to its action on serotonin $5-\mathrm{HT}_{2 \mathrm{~A}}$ 
and 5- $\mathrm{HT}_{2 \mathrm{C}}$ receptors (Arnt 1998; Glaser et al. 1998), and it has long been suggested that typical drugs can be made essentially atypical by careful dosing (Kapur et al. 1996). Another criterion of atypicality is the lower incidence of EPSE at therapeutic doses. The atypical antipsychotics olanzapine and risperidone do not usually cause EPSE at their threshold doses and infrequently cause EPSE at their dose of near-maximal effect (Davis and Chen 2004; Bishara et al. 2013), but do produce significant EPSE at daily doses above $20 \mathrm{mg}$ and $6 \mathrm{mg}$ respectively. EPSEs were frequently reported when flupentixol was given at a dose of $40 \mathrm{mg}$ every 2 weeksour proposed near-maximal effect dose. The proportion of patients who were prescribed anticholinergic medicines at therapeutic doses was similar to that seen in haloperidoltreated patients in a recent head-to-head comparison study with paliperidone (McEvoy et al. 2014). So, even at the doses suggested here, flupentixol remains effectively a typical antipsychotic.

\section{Optimal dosing and recommendation for clinical practice}

The optimal dose is the dose of drug that will produce the desired effect with the least likelihood of undesirable effects. The only study that aimed to find the optimal dose of flupentixol decanoate in the maintenance treatment of schizophrenia found that individualised doses between $5 \mathrm{mg}$ and $40 \mathrm{mg}$ every 1 to 4 weeks was the most successful (Pach et al. 1998). In this review, we found high success rates across the studied dose range. Relapse prevention for a chronic illness such as schizophrenia is desirable over a long period of time, and psychotic exacerbations may not be seen until over 12 months after a dose reduction (Marder et al. 1987). The relatively short period of 6 months may therefore not be long enough to estimate the dose that will prevent relapses in the longer term, although most of our included studies were longer than 6 months.

We found that rates of EPSE did not correlate with dose, but these were only reported in studies with mean doses of $40.7 \mathrm{mg}$ every 2 weeks or less. Even at these doses, EPSEs were seen frequently. From this, we estimate the optimal dose of flupentixol decanoate in the maintenance treatment of schizophrenia to be between $20 \mathrm{mg}$ and $40 \mathrm{mg}$ every 2 weeks. We acknowledge the relative inconvenience of two weekly dosing and suggest that injection intervals should be individually established.

The average apparent half-life of flupentixol decanoate is 17 days, leading to a time to steady state of around 2 months. Effects of initial dose and subsequent dose changes on plasma levels will therefore take 2 months to become fully apparent. This delay makes dose titration difficult, and clinicians may be tempted to increase doses before the drug has reached steady state. Stabilisation with the more flexible oral flupentixol in the acute psychotic state is therefore preferable because dose can be more closely related to effects.

When switching to the long-acting injectable preparation, we suggest a starting dose of $20 \mathrm{mg}$ every 2 weeks, which may be increased after 8 weeks to $30 \mathrm{mg}$ or $40 \mathrm{mg}$ every 2 weeks if necessary. For some patients, a lower dose of $10 \mathrm{mg}$ every 2 weeks may be sufficient, and other patients may require higher doses. Doses above $100 \mathrm{mg}$ every 2 weeks should only be given in exceptional circumstances, if tolerability allows.

For patients currently established on doses above $100 \mathrm{mg}$ every 2 weeks, a cautious down-titration of dose may be warranted to reduce risk of tardive dyskinesia. The reviewed studies suggest that halving the dose in a previously stable patient puts them at danger of deterioration. It would therefore be pragmatic to reduce patients' doses in small decrements with sufficient time between decrements to monitor the effect.

\section{Strengths and limitations}

This review expands on the work by Reed and Fanshawe (2011) as it contains additional data from German-language studies, non-RCTs and the study by Dencker and co-workers (1980) which used flupentixol palmitate. This provided us with over twice as many patients as the review from 2011. We standardised treatment success rates to 6 months to account for time as a factor influencing relapse.

The included RCTs were small, making it harder to generalise the results. The larger, non-comparator studies had other limitations: e.g. the study by Chiliza et al. (2016) was primarily a feasibility and acceptability study and included firstepisode patients only, and the study by Shajahan et al. (2010) included mostly middle-aged, Caucasian patients.

By standardising treatment failure rates to 6 months, we risk underestimating relapse rates. The longest studies (Gottfries and Green 1974; Wistedt and Ranta 1983) had the highest treatment failure rates over the entire study period but did report on relapse and dropouts at 6 months. For seven studies, treatment failure rates needed to be calculated, assuming a linear relapse rate. The study by Kane et al. (2012) demonstrated a linear relapse rate over the first 6 months for patients on treatment with aripiprazole longacting injection, with relapses becoming less frequent after 6 months, but continuing at a linear rate for placebo-treated patients. Similar time-to-relapse plots were seen in the MARS study (Pach et al. 1998) and in the study by Shajahan et al. (2010).

Using mean doses as single data points to plot the doseresponse curve can prove problematic, as they would more accurately be displayed as horizontal lines. Data from the fixed dose studies generally fit the curve, but do not provide relapse data beyond a dose of $26.67 \mathrm{mg}$ every 2 weeks. 


\section{Conclusion}

This review suggests that the optimal dose of flupentixol decanoate in the maintenance treatment of schizophrenia is likely to be between 20 and $40 \mathrm{mg}$ every 2 weeks, although some patients may be able to remain well on lower doses or on dosing intervals of 3 or 4 weeks. We suggest that doses of flupentixol should be individually established in the range of 10 to $40 \mathrm{mg}$ every 2 weeks according to response and tolerability. Given pharmacokinetic variability, some patients may benefit from higher doses, but this should be the exception. Patients already established on higher doses may deteriorate if their dose is drastically or too quickly reduced. Dose reductions should therefore only take place after careful consideration of risks. Our suggested dose range is more in line with the product specifications of Fluanxol®, the brand under which flupentixol decanoate is marketed in countries outside of the UK and Ireland. We recommend that the Depixol® product label be revised to closer resemble that of Fluanxol®, which is more consistent with the evidence, as demonstrated in this review.

Acknowledgments The authors thank King's College London for making this article open access.

\section{Compliance with ethical standards}

Conflict of interest D.T. has received payments for lectures and advisory boards from Janssen, Lundbeck, Sunovion and Otsuka. L.B. declares no conflict of interest in preparing this article.

Open Access This article is distributed under the terms of the Creative Commons Attribution 4.0 International License (http:// creativecommons.org/licenses/by/4.0/), which permits unrestricted use, distribution, and reproduction in any medium, provided you give appropriate credit to the original author(s) and the source, provide a link to the Creative Commons license, and indicate if changes were made.

\section{References}

Aaes-Jorgensen T (1980) Specific high-performance liquid chromatographic method for estimation of the cis(Z)- and trans(E)-isomers of clopenthixol and a N-dealkyl metabolite. J Chromatogr 183(2): 239-245

Agrup-Andersson L, Bengtsson A, Erlandsson K, Gottfries CG, WitzellOstlund G (1974) Flupenthixol decanoate - controlled investigation concerning dosage. Acta Psychiatr Scand 255(S):7-14

Arnt J (1998) Pharmacological differentiation of classical and novel antipsychotics. Int Clin Psychopharmacol 13(Suppl 3):S7-S14

Arvanitis LA, Miller BG (1997) Multiple fixed doses of "Seroquel" (quetiapine) in patients with acute exacerbation of schizophrenia: a comparison with haloperidol and placebo. The Seroquel Trial 13 Study Group. Biol Psychiatry 42(4):233-246

Balant-Gorgia AE, Eisele R, Aeschlimann JM, Balant LP, Garrone G (1985) Plasma flupentixol concentrations and clinical response in acute schizophrenia. Ther Drug Monit 7(4):411-414
Bishara D, Olofinjana O, Sparshatt A, Kapur S, Taylor D, Patel MX (2013) Olanzapine: a systematic review and meta-regression of the relationships between dose, plasma concentration, receptor occupancy, and response. J Clin Psychopharmacol 33(3):329-335

Chiliza B, Asmal L, Oosthuizen P, Emsley R, Kidd M, Ojagbemi A, Esan O, Gureje O (2016) Combining depot antipsychotic with an assertive monitoring programme for treating first-episode schizophrenia in a resource-constrained setting. Early Intervent Psychiatry 10(1): $54-62$

Chouinard G, Jones BD, Annable L (1978) Neuroleptic-induced supersensitivity psychosis. Am J Psychiatry 135(11):1409-1410

Cookson IB (1987) The effects of a 50\% reduction of cis(z)-flupenthixol decanoate in chronic schizophrenic patients maintained on a high dose regime. Int Clin Psychopharmacol 2(2):141-149

Creese I, Snyder SH (1977) A simple and sensitive radioreceptor assay for antischizophrenic drugs in blood. Nature 270(5633):180-182

Davis JM, Chen N (2004) Dose response and dose equivalence of antipsychotics. J Clin Psychopharmacol 24(2):192-208

Dencker SJ, Lepp M, Malm U (1980) Clopenthixol and flupenthixol depot preparations in outpatient schizophrenics. I. A one year double-blind study of clopenthixol decanoate and flupenthixol palmitate. Acta Psychiatr Scand 279(S):10-28

Ezewuzie N, Taylor D (2006) Establishing a dose-response relationship for oral risperidone in relapsed schizophrenia. J Psychopharmacol (Oxford, England) 20(1):86-90

Farde L, Wiesel FA, Nordstrom AL, Sedvall G (1989) D1- and D2dopamine receptor occupancy during treatment with conventional and atypical neuroleptics. Psychopharmacology 99(Suppl):S28 S31

Finkbeiner T, Hagen S, Pach J, Tegeler J, Glaser T, Osterheider M (1998) Vertraglichkeit von flupentixol-decanoat in der rezidivprophylaxe schizophrener storungen-analyse der nebenwirkungen verschiedener dosierungsstrategien (MARS-studie) - tolerability of flupenthixol decanoate in prophylactic treatment of schizophrenic patients in remission - analysis of dose related side effects (MARS study). Psychopharmakotherapie 5(4):156-160

Glaser T, Sommermeyer M, Fassbinder M and Mauler F, 1998. Receptor binding of flupenthixol and other neuroleptics [Das Rezeptorbindungsprofilvon cis-Flupentixol]. In: T. Glaser and M. Soyka, eds, Flupentixol. Typisches oder Atypisches Wirkspektrum. Darmstadt, Germany: Steinkopff, pp. 6-21

Gottfries CG, Green L (1974) Flupenthixol decanoate-in treatment of out-patients. Acta Psychiatr Scand 255(S):15-24

Johnson DAW, Ludlow JM, Street K, Taylor RDW (1987) Double-blind comparison of half-dose and standard-dose flupenthixol decanoate in the maintenance treatment of stabilised out-patients with schizophrenia. Br J Psychiatry 151:634-638

Jørgensen A, Overø KF (1980) Clopenthixol and flupenthixol depot preparations in outpatient schizophrenics: III. Serum levels. Acta Psychiatr Scand 279:41-54 61(S)

Jorgensen A (1978) A sensitive and specific radioimmunoassay for cis (Z)-flupenthixol in human serum. Life Sci 23(15):1533-1542

Kane JM, Eerdekens M, Lindenmayer JP, Keith SJ, Lesem M, Karcher K (2003) Long-acting injectable risperidone: efficacy and safety of the first long-acting atypical antipsychotic. Am J Psychiatry 160(6): $1125-1132$

Kane JM, Sanchez R, Perry PP, Jin N, Johnson BR, Forbes RA, Mcquade RD, Carson WH, Fleischhacker WW (2012) Aripiprazole intramuscular depot as maintenance treatment in patients with schizophrenia: a 52-week, multicenter, randomized, double-blind, placebocontrolled study. J Clin Psychiatry 73(5):617-624

Kapur S, Remington G, Jones C, Wilson A, Dasilva J, Houle S, Zipursky R (1996) High levels of dopamine D2 receptor occupancy with lowdose haloperidol treatment: a PET study. Am J Psychiatry 153(7): 948-950 
Kelly HB, Freeman HL, Banning B, Schiff AA (1977) Clinical and social comparison of fluphenazine decanoate and flupenthixol decanoate in the community maintenance therapy of schizophrenia. Int Pharmacopsychiatry 12:54-64

Kistrup K, Gerlach J, Aaes-Jorgensen T, Larsen N (1991) Perphenazine decanoate and $\operatorname{cis}(\mathrm{Z})$-flupentixol decanoate in maintenance treatment of schizophrenic outpatients. Serum levels at the minimum effective dose. Psychopharmacology 105(1):42-48

Knights A, Okasha MS, Salih MA, Hirsch SR (1979) Depressive and extrapyramidal symptoms and clinical effects: a trial of fluphenazine versus flupenthixol in maintenance of schizophrenic out-patients. $\mathrm{Br}$ J Psychiatry 135:515-523

Larsen NE, Hansen LB, Knudsen P (1985) Quantitative determination of perphenazine and its dealkylated metabolite using high-performance liquid chromatography. J Chromatogr 341(1):244-250

Laux G, Steinman C, Glaser T (2010) Long term treatment of schizophrenic psychoses with flupentixol decanoate: results of a noninterventional study. Psychopharmakotherapie 17:274-284

Leucht S, Tardy M, Komossa K, Heres S, Kissling W, Salanti G, Davis JM (2012) Antipsychotic drugs versus placebo for relapse prevention in schizophrenia: a systematic review and meta-analysis. Lancet (London, England) 379(9831):2063-2071

Lieberman JA, Stroup TS, Mcevoy JP, Swartz MS, Rosenheck RA, Perkins DO, Keefe RS, Davis SM, Davis CE, Lebowitz BD, Severe J, Hsiao JK, Clinical Antipsychotic Trials of Intervention Effectiveness (catie) Investigators (2005) Effectiveness of antipsychotic drugs in patients with chronic schizophrenia. N Engl J Med 353(12):1209-1223

Lundbeck LIMITED, (2017a)-last update, Depixol 20mg/ml solution for injection - summary of product characteristics (eMC). Available: https://www.medicines.org.uk/emc/product/997. Accessed 11 Aug 2018

Lundbeck LIMITED, (2017b)-last update, Depixol tablets 3mgsummary of product characteristics (eMC). Available: https:// www.medicines.org.uk/emc/product/997. Accessed 27 Feb 2018

Mahapatra, J., Quraishi, S.N., David, A., Sampson, S. and Adams, C.E., 2014. Flupenthixol decanoate (depot) for schizophrenia or other similar psychotic disorders. The Cochrane database of systematic reviews, (6):CD001470

Marder SR, Van Putten T, Mintz J, Lebell M, Mckenzie J, May PR (1987) Low- and conventional-dose maintenance therapy with fluphenazine decanoate. Two-year outcome. Arch Gen Psychiatry 44(6): $518-521$

Mccreadie R, Wiles D, Grant S, Moore J, Crocket G, Mahmood Z, Livingston M, Watt J, Greene J, Kershaw P, Todd N, Scott A, Loudon J, Dyer J, Philip A, Batchelor D (1988) The Scottish first episode schizophrenia study V. one-year follow-up. The Scottish Schizophrenia Research Group. Br J Psychiatry : the journal of mental science 152:470-476

Mcevoy JP, Byerly M, Hamer RM, Dominik R, Swartz MS, Rosenheck RA, Ray N, Lamberti JS, Buckley PF, Wilkins TM, Stroup TS (2014) Effectiveness of paliperidone palmitate vs haloperidol decanoate for maintenance treatment of schizophrenia: a randomized clinical trial. Jama 311(19):1978-1987

Pach J, Bertling R, Finkbeiner T, Haug HJ, Knoche M, Osterheider M, Reinertshofer T, Tegeler J (1998) Rezidive bei schizophrenen erkrankungen unter depot-neuroleptika-Ergebnisse einer einjahrigen doppelblindstudie mit flupentixol-decanoat-relapse in schizophrenia under maintenance depot neuroleptic therapy- results of a 1-year double-blind study with flupenthixol decanoate. Psychopharmakotherapie 5(4):161-165

Pandina GJ, Lindenmayer JP, Lull J, Lim P, Gopal S, Herben V, Kusumakar V, Yuen E, Palumbo J (2010) A randomized, placebocontrolled study to assess the efficacy and safety of 3 doses of paliperidone palmitate in adults with acutely exacerbated schizophrenia. J Clin Psychopharmacol 30(3):235-244

Pinto R, Bannerjee A, Ghosh N (1979) A double-blind comparison of flupenthixol decanoate and fluphenazine decanoate in the treatment of chronic schizophrenia. Acta Psychiatr Scand 60(4):313-322

Reed P, Fanshawe T (2011) The effects of dose of flupentixol decanoate on relapse rates in schizophrenia. Psychiatrist 35(8):293-296

Reimold M, Solbach C, Noda S, Schaefer JE, Bartels M, Beneke M, Machulla HJ, Bares R, Glaser T, Wormstall H (2007) Occupancy of dopamine $\mathrm{D}(1), \mathrm{D}(2)$ and serotonin (2A) receptors in schizophrenic patients treated with flupentixol in comparison with risperidone and haloperidol. Psychopharmacology 190(2):241-249

Saikia JK, Jørgensen A (1983) Steady-state serum concentrations after cis (Z)-flupentixol decanoate in viscoleo. Psychopharmacology 80(4): 371-373

Shajahan P, Daniel D, Pelosi A, Taylor M, Spence E (2010) Comparison of the effectiveness of depot antipsychotics in routine clinical practice. Psychiatrist 34(7):273-279

Steinert J, Erba E, Pugh CR, Robinson C, Priest RG (1986) A comparative trial of depot pipothiazine. J Int Med Res 14:72-77

Taylor D, Barnes TR, Young A (2018) The Maudsley prescribing guidelines in psychiatry, 13th edn. John Wiley \& Sons Inc, Chichester, West Sussex ; Hoboken, NJ

Taylor DM, Young C, Patel MX (2006) Prospective 6-month follow-up of patients prescribed risperidone long-acting injection: factors predicting favourable outcome. Int J Neuropsychopharmacol 9(6): 685-694

Taylor D (2005) Establishing a dose-response relationship for haloperidol decanoate. Psychiatr Bull 29(3):104-107

Turbott J, Villiger J, Hunter L (1985) Neuroleptic serum levels measured by radioreceptor assay in patients receiving intramuscular depot neuroleptics. Some preliminary findings. Br J Psychiatry 146:439442

Uchida H, Suzuki T, Takeuchi H, Arenovich T, Mamo DC (2011) Low dose vs standard dose of antipsychotics for relapse prevention in schizophrenia: meta-analysis. Schizophr Bull 37(4):788-799

Wistedt B (1981) A depot neuroleptic withdrawal study. A controlled study of the clinical effects of the withdrawal of depot fluphenazine decanoate and depot flupenthixol decanoate in chronic schizophrenic patients. Acta Psychiatr Scand 64(1):65-84

Wistedt B, Jørgensen A, Wiles D (1982) A depot neuroleptic withdrawal study. Plasma concentration of fluphenazine and flupenthxiol and relapse frequency. Psychopharmacology 78:301-304

Wistedt B, Ranta J (1983) Comparative double-blind study of flupenthixol decanoate and fluphenazine decanoate in the treatment of patients relapsing in a schizophrenic symptomatology. Acta Psychiatr Scand 67(6):378-388

World Health Organisation, 2018-last update, WHOCC - Definition and general considerations. Available: https:/www.whocc.no/ddd/ definition_and_general_considera/. Accessed 3 Mar 2018]

Publisher's note Springer Nature remains neutral with regard to jurisdictional claims in published maps and institutional affiliations. 